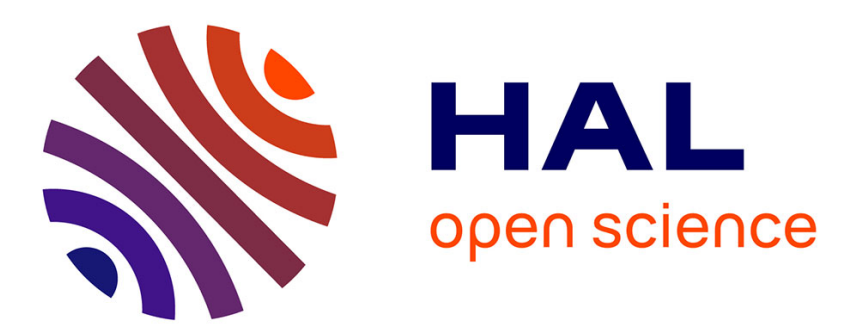

\title{
Handing off iron to the next generation: how does it get into seeds and what for?
}

Stéphane Mari, Christophe Bailly, Sébastien Thomine

\section{To cite this version:}

Stéphane Mari, Christophe Bailly, Sébastien Thomine. Handing off iron to the next generation: how does it get into seeds and what for?. Biochemical Journal, 2020, 477 (1), pp.259-274. 10.1042/BCJ20190188 . hal-02445553

\section{HAL Id: hal-02445553 https://hal.science/hal-02445553}

Submitted on 14 Dec 2020

HAL is a multi-disciplinary open access archive for the deposit and dissemination of scientific research documents, whether they are published or not. The documents may come from teaching and research institutions in France or abroad, or from public or private research centers.
L'archive ouverte pluridisciplinaire HAL, est destinée au dépôt et à la diffusion de documents scientifiques de niveau recherche, publiés ou non, émanant des établissements d'enseignement et de recherche français ou étrangers, des laboratoires publics ou privés. 
1 title

2 Handing off iron to the next generation: how does it get into seeds and

3 what for?

4

Stéphane Mari ${ }^{1}$, Christophe Bailly ${ }^{2}$, Sébastien Thomine ${ }^{3 *}$

6

${ }^{1}$ BPMP, INRA, CNRS, Univ Montpellier, Montpellier SupAgro, Montpellier, France

$8{ }^{2}$ Sorbonne Université, CNRS, Laboratoire de Biologie du Développement, F-75005 Paris,

9 France

$10{ }^{3}$ Institute for Integrative Biology of the Cell (I2BC), CEA, CNRS, Univ. Paris-Sud,

11 Université Paris-Saclay, 91198, Gif-sur-Yvette cedex, France

12 *to whom correspondence should be addressed

$13 \quad$ Sebastien.thomine@i2bc.paris-saclay.fr

$14+33169824632$

\section{Abstract}

To ensure the success of the new generation in annual species, the mother plant transfers a large proportion of the nutrients it has accumulated during its vegetative life to the next generation through its seeds. Iron $(\mathrm{Fe})$ is required in large amounts to provide the energy and redox power to sustain seedling growth. However, free Fe is highly toxic as it leads to the generation of reactive oxygen species. Fe must therefore be tightly bound to chelating molecules to allow seed survival for long periods of time without oxidative damage. Nevertheless, when conditions are favorable, the seed Fe stores have to be readily remobilized to achieve the transition towards active photosynthesis before the seedling becomes able to take up Fe from the environment. This is likely critical for the vigor of the young plant. Seeds constitute an important dietary source of Fe, which is essential for human health. Understanding the mechanisms of Fe storage in seeds is key to improve their Fe content and availability in order to fight the Fe deficiency. Seed longevity, germination efficiency and seedling vigor are also important traits that may be affected by the chemical form under which $\mathrm{Fe}$ is stored. In this review, we summarize the current knowledge on seed Fe loading during development, long-term storage and remobilization upon germination. We highlight how this knowledge may help seed Fe biofortification and discuss how Fe storage may affect seed quality and germination efficiency. 


\section{Introduction}

Seed is a very special stage in the life of a plant. It is the basis for the next generation and the survival of the species. To ensure the success of the new generation, the mother plant transfers a large proportion of the nutrients it has accumulated during its vegetative life to its seeds. Most work on seed nutrient storage focuses on carbohydrate, lipids and proteins, which provide the bulk energy, carbon and nitrogen required to initiate development. Depending on species, 30 to $90 \%$ of plant nitrogen is allocated to seeds at the end of the plant life cycle ${ }^{1}$. However, mineral stores are also very important to sustain seedling growth. Especially, iron $(\mathrm{Fe})$ is required in large amount in mitochondria, which provide the energy and redox power during the initial stage of seedling development. For example, in the case of Arabidopsis (Arabidopsis thaliana), 50 to $55 \%$ of the iron $(\mathrm{Fe})$ is allocated to seeds at the end of the life cycle $^{2,3}$. Moreover, the nutrients need to be stored in a compact and stable form. Seed storage proteins form pseudo crystalline aggregates in protein bodies within seed embryo cells. Free $\mathrm{Fe}$ is highly toxic as it leads to the generation of reactive oxygen species through the Fenton reaction. In cells, Fe must therefore be tightly bound to chelating molecules to prevent the formation of ROS. This is probably even more important in seeds than in other organs, as seeds represent resistance forms designed to survive for long periods of time, often several years, and through stresses. Nevertheless, when conditions are favorable, the nutrients stored in seeds have to be readily remobilized to sustain the early development of the seedling before it acquires photosynthetic capacity and becomes able to take up nutrients from the environment. Iron is a major component of photosystem I and its availability to the young seedling is necessary to achieve the transition towards active photosynthesis. This is likely critical for the vigor of the young plant.

From the point of view of animals and especially human beings, seeds are a major food resource. They provide carbohydrates, proteins and fat but also micronutrients such as $\mathrm{Fe}$ and zinc, which are essential for human health. About 2 billion human beings suffer from $\mathrm{Fe}$ deficiency, which leads to asthenia, anemia and in extreme cases to death. The prevalence of Fe deficiency is highest in populations that rely mostly on a plant based diet because plants and seeds in particular do not provide sufficient $\mathrm{Fe}^{4}$. Understanding the mechanisms of $\mathrm{Fe}$ storage in seeds is key to improve their Fe content and availability in order to fight $\mathrm{Fe}$ deficiency using biofortification. From the agricultural perspective, seed longevity, germination efficiency and seedling vigor are also very important traits that breeders and 
chemical form of Fe may be critical. In this review, we will summarize the current knowledge on seed Fe loading during development, long-term storage and remobilization upon germination, updating the last review on the topic ${ }^{5}$. We will highlight how this knowledge may help seed Fe biofortification, longevity, germination efficiency and stand establishment.

\section{Iron uptake and translocation to the shoots}

Plants take up iron using distinct strategies according to species ${ }^{6,7}$. Most plant species use strategy I, which is based on the reduction of Fe in the rhizosphere followed by its uptake by a transporter for $\mathrm{Fe}(\mathrm{II})$. In contrast, graminaceous species, which include the major grain crops such as rice, wheat and barley, have evolved strategy II. Strategy II is based on the release of small $\mathrm{Fe}(\mathrm{III})$ chelating molecules called phytosiderophores in the rhizosphere and subsequent uptake of siderophore-Fe(III) complexes. Under Fe deficiency, strategy I plants activate the expression of the genes involved in Fe solubilization and uptake. The molecular players in this response have been identified in Arabidopsis but are essentially conserved in other species. The proton pump Arabidopsis plasma membrane $\mathrm{H}^{+}$-ATPase 2 (AHA2) drives rhizosphere acidification which is important to solubilize $\mathrm{Fe}^{8}$. The coumarin transporter Pleiotropic Drug Resistance 9 (PDR9) allows the secretion of coumarin with iron binding and reducing properties in the rhizosphere. Enzymes of the coumarin biosynthetic pathway are induced along with $P D R 9^{9,10}$. The membrane bound Ferric chelate Reductase FRO2 reduces chelate-bound $\mathrm{Fe}(\mathrm{III})$ to $\mathrm{Fe}(\mathrm{II})$, which is taken up into root cells by the divalent metal Iron Regulated Transporter 1 (IRT1) ${ }^{11,12}$. IRT1 is not specific for Fe(II) and drives the uptake of a range of other divalent metal cations including $\mathrm{Zn}, \mathrm{Mn}, \mathrm{Co}, \mathrm{Cd}, \mathrm{Ni}{ }^{13-15}$. A sophisticated mechanism enables the removal of IRT1 from the plasma membrane when intracellular concentrations of non-Fe metals raise ${ }^{16}$. In addition, several genes involved in the sequestration of these metal cations are activated under Fe deficiency ${ }^{17}$. In graminaceous species such as rice, maize and barley, the components that are upregulated for Fe acquisition under $\mathrm{Fe}$ deficiency include the phytosiderophore efflux and Fe-sirerophore influx transporters as well as the biosynthetic pathway of phytosiderophores of the mugineic acid family ${ }^{7}$. The $\mathrm{S}$-adenosyl methionine cycle is activated to produce methionine, which is used by the nicotianamine synthase (NAS) to produce nicotianamine (NA) the precursor of mugineic acids. Whereas NA is also synthetized in strategy I species, mugineic acids are unique to graminaceous species. NA is converted to mugineic acid by the nicotianamine aminotransferase (NAAT) and to deoxymugineic acid by the deoxymugineic acid synthase (DMAS). MA and DMA are secreted to the rhizosphere by an efflux transporter, Transporter 
102 Of Mugineic acid 1 (TOM1) in rice (Oryza sativa), Yellow Stripe 3 (YS3) in maize (Zea

103 mais) ${ }^{18,19}$. Fe-siderophore complexes are taken up by specific influx transporters, Yellow

104 Stripe 1 (YS1) in maize and YS1-Like 15 (YSL15) in rice ${ }^{20-22}$. Despite the difference in their

105 uptake strategies, the control of Fe deficiency responses involves a similar network of

106 negatively and positively regulating bHLH (basic Helix Loop Helix) in graminaceous plants

107 and the other species ${ }^{23,24}$. In addition, Hemerythrin Domain containing RING proteins that

108 act as negative regulators of $\mathrm{Fe}$ deficiency responses by mediating the $\mathrm{Fe}$ dependent

109 degradation of specific bHLH, are also conserved among strategy I and strategy II plants ${ }^{25-27}$.

110 To be translocated to the shoots, Fe has to go through the endodermis cell layer and to be

111 released into the xylem sap together with ligands that maintain it in soluble form. Recently,

112 suberin deposition around the endodermis has been shown to provide a control point for Fe

113 translocation. Under Fe deficiency, the suberin layer is degraded allowing the transfer of Fe

114 from the root cortex to the central cylinder where it can be loaded into the xylem for

115 translocation to the aerial parts ${ }^{28,29}$. Based on its expression pattern and plasma membrane

116 localization, the Fe efflux transporter Ferroportin (FPN1)/Iron REGulated 1 (IREG1) has been

117 proposed to mediate Fe loading in the xylem in Arabidopsis ${ }^{30}$. The MATE family citrate

118 efflux transporters, Ferric Reductase Deficient 3 (FRD3) in Arabidopsis and FRD3- Like

119 1(FRDL1) in rice, play an important role in the translocation of Fe and other metals, by

120 loading citrate which chelates Fe and prevent its precipitation in the xylem sap ${ }^{31-35}$.

\section{Remobilization from other organs to the seeds}

123 At the vegetative stage, most of the iron translocated to aerial parts is used for photosynthesis

124 in chloroplasts in mesophyll cells. Photosystem I and Ferredoxin, which are very abundant in 125 chloroplasts, contain numerous FeS clusters. This assumption based on biochemical needs is 126 supported by cell fractionation experiments and Fe imaging in leaves ${ }^{36-38}$. Upon transition to 127 the reproductive stage, a large part of the Fe present in vegetative tissues is transferred to the 128 seeds. In Arabidopsis, 50 to 55\% of total plant Fe ends up in the seeds at the end of the life 129 cycle (the Iron Harvest Index, Figure 1) ${ }^{2,3}$. A recent study based on Fe isotope labeling 130 indicated that most of the Fe stored in seeds originates from vegetative tissue, implying a 131 major remobilization of $\mathrm{Fe}^{2}$.

132 Prior to its movement from the vegetative tissues to the seeds, Fe has to be made available in 133 the senescing tissues. Autophagy was proposed to play a key role in nutrient remobilization 134 from vegetative tissues to seeds ${ }^{39}$. This was based on the finding that autophagy is involved 135 in nitrogen remobilization to seeds ${ }^{40}$. Recently, the finding that a mutation in Autophagy 5 
136 (ATG5), which strongly impairs autophagy, decreases the proportion of Fe allocated to seeds 137 from $50-55 \%$ to $15-20 \%$ highlighted the importance of autophagy for Fe remobilization ${ }^{2}$. 138 Similar results were obtained for other micronutrients such as $\mathrm{Zn}$ and $\mathrm{Mn}$. The role of 139 autophagy is thus clearly not limited to Fe. Recently, several pathways have been implicated 140 in the degradation of chloroplasts, which contain most of the cellular Fe, during leaf 141 senescence ${ }^{41}$. They include (i) the senescence-associated vacuoles (SAV) that are important 142 for the degradation of stromal proteins such as RuBisCo and glutamine synthase, (ii) 143 chlorophagy in which whole chloroplast are encapsulated in autophagosomes ${ }^{42}$ (iii) a 144 vesicular pathway depending on the ATG8 Interacting protein (ATI) which degrades envelope and thylakoid membranes in addition to stromal proteins ${ }^{43}$ and (iv) $\mathrm{CV}$-Containing vesicles

146 (CCVs) that contain the CV protein (Chloroplast Vesiculation) responsible for chloroplast 147 destabilization ${ }^{44}$. In contrast to the others, the CCV pathway does not require autophagy. It 148 will be interesting to determine the respective importance of these pathways for $\mathrm{Fe}$ 149 remobilization during vegetative organ senescence, as they could allow to target some 150 nutrients more specifically than autophagy.

151 In addition to autophagy, the timing of senescence is also an important factor controlling remobilization. If senescence occurs too fast, the time window for remobilization is reduced and less nutrients are allocated to seeds. Hence, NAC transcription factors, which control the onset of senescence, also play a major role in the control of nutrient remobilization from leaves to seeds ${ }^{45-47}$. More work will be required to understand how the interplay between autophagy and senescence controls Fe allocation to seeds in different species.

157 After it has been made available in vegetative tissue, Fe has to be transported to developing seeds through the phloem. Many lines of evidence have pointed to a key role of NA in this process. Complete loss of NA synthesis leads to sterility ${ }^{48-50}$. This has initially prevented a direct analysis of the role of NA in Fe transport to seeds, as the mutants did not set seeds. The first evidence that NA is important for Fe transport to the seeds came from the study of mutants impaired in YS1-like genes (YSL). The strong expression of OsYSL2 in phloem tissues of rice aerial parts and developing seeds and the ability of this transporter to take up NA-Fe and NA-Mn complexes when expressed in Xenopus oocytes provided the first indication that

165 NA is important for Fe transport through the phloem to the seeds ${ }^{51}$. Analysis of rice lines in which OsYSL2 expression was silenced further supported this hypothesis ${ }^{52}$. In these lines, $\mathrm{Fe}$ transport to the shoots and the seeds was strongly decreased. Conversely, increased OSYSL2 expression under a phloem specific promoter led to strong increase in seed endosperm $\mathrm{Fe}$ concentration ${ }^{52}$. More recently, another YSL transporter from rice, OsYSL9, was implicated 
170 in Fe delivery to developing rice grain. OsYSL9 expression is upregulated by Fe deficiency in 171 roots but down regulated in leaves under the same conditions ${ }^{53}$. The role of YSL in metal 172 transport to seeds is not restricted to rice. Similar conclusions were obtained from the analysis 173 of Arabidopsis knockout mutants targeting AtYSL1 gene or double mutants affecting AtYSL1 174 and AtYSL3 ${ }^{54-56}$. Like OsYSL2, AtYSL1 is expressed in phloem tissues of leaves as well as in 175 developing siliques. Similar to OSYSL9, it is down regulated under Fe deficiency. Loss of 176 function mutants in AtYSL1 accumulated higher levels of NA in shoots but strongly decreased 177 NA and Fe concentrations in mature seeds ${ }^{55}$. Combining a mutation in AtYSLl with a 178 mutation in another Arabidopsis YSL strongly expressed in phloem, AtYSL3, leads to more severe phenotypes: the ysllysl3 mutants display symptoms of strong $\mathrm{Fe}$ deficiency with interveinal chlorosis as well as decreased fertility. Like ysll, they also accumulate lower concentrations of $\mathrm{Fe}$ in their seeds when provided with a high $\mathrm{Fe}$ concentration in the medium, which alleviates the defect in fertility and up-regulate $Y S L 1$ expression ${ }^{55,56}$. Further inflorescence grafting experiments showed that expression of AtYSL1 and AtYSL3 in the rosette was sufficient to restore fertility but not seed Fe content ${ }^{54}$. Furthermore, the identification of a quadruple mutant combining knockout in 3 out of the 4 genes encoding NAS in Arabidopsis and a knockdown in the fourth one allowed to obtain fertile plants with drastically decreased levels of NA ${ }^{48}$. In this quadruple nas mutant, Fe and NA levels are lower in seeds, similar to the phenotype observed in ysll. Interestingly, in NA biosynthesis mutants, Fe accumulates in phloem cells indicating that NA is required to retrieve Fe from the phloem and provide it to sink tissues, such as seeds ${ }^{57}$. This role may be shared between NA and mugineic acids in graminaceous plants. On the other hand, all YSL transporters characterized so far using expression in yeast or in Xenopus oocytes mediate the influx of NA-Fe or MA-Fe complexes into cells. The finding that NA and YSL are important for phloem transport of Fe does not imply that Fe is transported as NA-Fe complex in the phloem. Another important player in Fe transport from vegetative organs to the seeds is the oligopeptide transporter OligoPeptide Transporter 3 (OPT3). Full loss of function of OPT3 leads to embryo lethality suggesting that the substrate of OPT3 is an essential cellular metabolite ${ }^{58}$. Even though the closest homologue of OPT3, BjGT1, is a glutathione transporter ${ }^{59}$, transport assays in yeast failed to show OPT3 ability to transport GSH ${ }^{60}$. Instead, expression of OPT3 could complement the yeast fet $3 f e t 4$ mutant impaired in $\mathrm{Fe}$ uptake, suggesting that it transports Fe complexes. The exact substrate of OPT3 remains to be identified. The identification of a weak allele of opt3, opt3-2 allowed investigating its function at the reproductive stage ${ }^{61}$. In opt3-2 mutants, Fe concentration is increased in 
vegetative organs but decreased in seeds, indicating a role in $\mathrm{Fe}$ transfer from vegetative organs to seeds. More recently, OPT3 was shown to be targeted to the plasma membrane and mainly expressed in phloem cells ${ }^{60,62}$. In opt3-2 mutants, Fe concentration was strongly increased in the xylem sap and decreased in the phloem sap, pointing to role of OPT3 in Fe transfer from the xylem to the phloem ${ }^{60}$. Combined with its expression in phloem cells, this suggests that OPT3 is involved in Fe loading into the phloem. Hence, both OPT3 and NA play critical roles in long distance transport of $\mathrm{Fe}$ in the phloem to sink organs and especially seeds: OPT3 would be necessary for loading Fe into the phloem, while NA would be required for Fe retrieval from the phloem ${ }^{57,60}$. Interestingly, OPT3 is also important for Fe deficiency signaling and opt3-2 up-regulated Fe-deficiency responsive gene expression even under $\mathrm{Fe}$ sufficient conditions ${ }^{60,62}$. In rice, OsOPT7, a homologue of OPT3, was characterized ${ }^{63}$. OsOPT7 could not transport GSH or Fe when expressed in Xenopus oocytes and yeast. Like AtOPT3, OsOPT7 is induced under Fe deficiency but its expression is more widespread than that of $O P T 3^{61,63}$. Moreover, the phenotype of opt7 mutant does not resemble that of opt3-2. Whether graminaceous plant genomes carry orthologues of OPT3 remains to be determined.

\section{Iron loading into seed tissues}

There is no continuity between the vasculature of the mother plant and the pro-vasculature of the embryo. Therefore, Fe, as other nutrients, has to be released from the phloem and re absorbed by the embryo. In Arabidopsis and other dicotyledonous plants, nutrients are released at the level of the chalaze and the nucellus into the embryo sac fluid from which the embryo takes up nutrients (Figure 2). Accordingly, AtYSL1 expression is detected in the funiculus and the chalazal endosperm ${ }^{55}$. Analyses of Fe speciation in the embryo sac fluid of developing pea seeds revealed that $\mathrm{Fe}$ is present as ferric $\mathrm{Fe}$ bound to citrate and malate in this extracellular compartment ${ }^{64}$. In agreement with this finding, the citrate efflux transporter FRD3 is expressed in the peripheral cell layer of the embryo and the cell layer of the tegument facing the embryo sac during seed development (Figure 2$)^{34}$. This suggest that FRD3 secretes citrate in the embryo sac to maintain Fe solubility and availability for uptake by the embryo.

232 In dicotyledonous plants, Fe is taken up as ferrous Fe. This implies that Fe must be reduced prior to uptake by the embryo. However, genetic analyses in Arabidopsis failed to identify a membrane bound ferric chelate reductase that is important for Fe acquisition by the embryo among FRO2 homologues. Instead, further analysis of the embryo sac fluid in pea showed the presence of a high concentration of ascorbate, sufficient to reduce $\mathrm{Fe}(\mathrm{III})$ to $\mathrm{Fe}(\mathrm{II})$ prior to its 
ascorbate biosynthesis, the seed Fe content is decreased. The speciation of Fe in the embryo

239 sac is thus similar to that encountered in other extracellular compartments of the plant, such as the xylem sap. In contrast, it is striking that ascorbate is used for Fe reduction, whereas membrane bound ferric chelate reductases are used for uptake in roots and leaves as well as in intracellular organelles such as mitochondria and plastids ${ }^{11,65,66}$. The transporters responsible

243 for secreting ascorbate and $\mathrm{Fe}$ in the embryo sac remain to be identified. In the case of $\mathrm{Zn}$, the 244 Heavy Metal pumping P-type ATPases HMA2 and HMA4 release Zn from the mother tissues 245 for subsequent uptake by the embryo ${ }^{67}$. In the case of $\mathrm{Fe}$, the plasma membrane $\mathrm{Fe}$ efflux 246 transporter IREG1/FPN1 may be involved in this process but so far no defect in Fe supply to 247 the embryo has been reported for iregl/fpnl mutant ${ }^{30}$.

248 In wheat and barley, the nutrients, including Fe, are provided to the grain by a single vascular 249 strand along the ventral crease ${ }^{68}$. Fe is mostly provided by the phloem and moves through 250 several specialized cell layers: the crease vascular parenchyma, the pigment strand and the 251 nucellar projection. Fe ends up in the transfer cells that are facing the embryo. From there, Fe 252 has to be released in the extracellular space separating the mother plant from the embryo 253 (Figure 3). The transfer cells, as the modified aleurone cells facing them on the side of the 254 embryo, have highly invaginated plasma membranes favoring nutrient release and reabsorption ${ }^{68}$. Fe accumulation and speciation in these structures show marked contrasts in the mature wheat grain. Fe is highly concentrated in the nucellar projection and co-localizes with sulfur. $\mathrm{Fe}$ accumulates to a lesser extend in the modified aleurone, from where it is probably distributed to the other aleurone cells, the embryo and the endosperm ${ }^{69}$. X-ray absorption spectra indicate that in the nucellar projection, Fe is mostly associated with NA, whereas in the modified aleurone it is associated with phytate ${ }^{69}$. In agreement with the speciation, barley homologues of AtVIT1, which drives Fe influx into the vacuole where phytate is localized, are strongly expressed in the aleurone ${ }^{68,70-72}$. In contrast, the genes encoding NAAT, NAS and YSL, that favor Fe mobility, are expressed at high levels in transfer cells ${ }^{68,73}$ (Figure 3). In the future, it will be important to determine the specific expression pattern and the role of each of the genes involved in Fe transport in this complex structure. This should allow the identification of the key transporters that release Fe to the extracellular space from the transfer cells as well as those responsible for taking it up into the embryo in the modified aleurone cells.

\section{Iron storage in seeds}


The localization of Fe in seeds differs according to species and seed developmental stage. The localization and subcellular localization are strongly associated with Fe speciation, i.e. the nature of the ligand that binds Fe and determines it bioavailability ${ }^{74}$. For example, Fe phytate complexes that are stored in vacuoles are notoriously poorly bioavailable ${ }^{75}$. In contrast, ferritin Fe stored in plastids constitutes a highly bioavailable source of $\mathrm{Fe}^{76}$.

Iron and other metals are not distributed evenly in seed tissues. In contrast, Fe distribution follows striking patterns. For example, in Arabidopsis mature embryo, $\mathrm{Fe}$ is highly concentrated around the vascular tissues ${ }^{71,77-81}$. Interestingly, the pattern of Fe distribution is distinct from that of other metals: $\mathrm{Mn}$ is concentrated in the sub-epidermal cell layers of Arabidopsis cotyledons in mature seeds while $\mathrm{Zn}$ is evenly distributed in Arabidopsis embryo $71,77,78,81$. The patterns of metal localization were initially discovered using synchrotron X-ray Fluorescence imaging of intact seeds and were then confirmed and refined using additional approaches. The use of Perls staining intensified by Diaminobenzidine (Perls DAB) allowed identification of the precise cell type accumulating $\mathrm{Fe}$ as the proto endodermis of the embryo ${ }^{80}$. The use of micro Particle Induced X-ray Emission ( $\mu$ PIXE) allowed quantification of the pattern: even though it is concentrated in proto-endodermal cells, $\mathrm{Fe}$ is present in other cells, albeit at much lower concentrations, and the Fe concentrated around pro-vascular tissues accounts for $50 \%$ of total seed Fe ${ }^{81}$. Finally, the use of Electron Dispersive X-ray imaging coupled to Transmission Electron Microscopy (TEM EDX) provided higher spatial resolution allowing the determination of the subcellular localization of $\mathrm{Fe}$ in the globoids of vacuoles of proto-endodermal cells (Figure 4) ${ }^{79,82}$. This subcellular localization is in agreement with the finding that mutations in AtVIT1 (Vacuolar Iron Transporter 1) disrupt the pattern of Fe distribution ${ }^{71,79}$. In vit1 mutants, $\mathrm{Fe}$ is not concentrated around vascular tissues but instead co-localizes with $\mathrm{Mn}$ in sub-epidermal cells ${ }^{71,79}$. Nevertheless, Fe is still localized in vacuoles in vit1 mutants ${ }^{79}$. Recently, the vacuolar Mn transporter AtMTP8 (Metal Tolerance Protein 8) was shown to be responsible for concentrating $\mathrm{Mn}$ in sub-epidermal cells in wild type seeds and Fe localization in these cells in the vit1 mutant background ${ }^{77,78}$. In $m t p 8$ knockout mutant seeds, $\mathrm{Mn}$ is concentrated around the vascular tissues together with Fe. In a double mutant combining vitl and mtp 8 mutations, $\mathrm{Mn}$ and Fe are evenly distributed in Arabidopsis embryo ${ }^{78}$. Therefore, by creating strong sinks for $\mathrm{Fe}$ or $\mathrm{Mn}$, vacuolar metal transporters determine the pattern of metal distribution in mature Arabidopsis embryo. The phenotype of vitl mutant, which survives poorly when germinated under Fe-deficient conditions, indicates that adequate tissue localization in the embryo is crucial ${ }^{71,79}$. Little information is available on how the Fe distribution pattern is generated during embryo 
development. Consistent with a strong expression of VIT1 during seed development, Fe patterning is already apparent at the torpedo stage at the center of the cotyledons where the provascular tissue will differentiate ${ }^{71,80}$. However, in Brassica napus, high resolution Perls $\mathrm{DAB}$ imaging detects $\mathrm{Fe}$ in the nuclei of all embryo cells at the torpedo stage ${ }^{83}$. Further during development, at the bent cotyledon stage, Fe is detected in cytoplasmic vesicles and finally ends up in the vacuoles of the cells surrounding the vasculature in mature embryo ${ }^{83}$. The observation of $\mathrm{Fe}$ in the nucleus at the torpedo stage in B. napus is consistent with the detection of high Fe concentrations in the nucleus of embryo cells in developing pea (pisum sativum) seeds ${ }^{84}$. Therefore, Fe subcellular localization evolves during embryo development. These changes in localization are most likely paralleled by changes in speciation and therefore of $\mathrm{Fe}$ bioavailability. The analysis of $\mathrm{Fe}$ bioavailability in pea seeds at different developmental stages show that Fe bioavailability in immature peas is higher than in mature pea seeds ${ }^{85}$.

Whether the pattern of Fe distribution in the vacuoles of cells surrounding the vasculature described in Arabidopsis is conserved among angiosperm has been investigated. In beans (Phaseolus vulgaris), Fe stores also concentrates around vascular tissues ${ }^{86}$. Recently, this observation was extended to Brassicales and even Rosids, which represent a wide group including a third of the angiosperms ${ }^{87,88}$. However, in many cases the number of cell layers that accumulate Fe is increased, to 2, the endodermis and one cortical cell layer in Brassicales, or even more cortical cell layers in other Rosids, compared to endodermis only in Arabidopsis ${ }^{83,87,88}$. While screening representatives of the different groups of angiosperm, interesting exceptions were identified in Chenopodium quinoa and Carica papaya ${ }^{87,88}$. There are certainly more diverse patterns to be discovered. In grains, the seeds of graminaceous species, the distribution of $\mathrm{Fe}$ is very different from that observed in Brassicales. In grains, in contrast to Arabidopsis, the embryo represents only a small volume of the seed, while the major part is constituted by the starchy endosperm. The endosperm is surrounded by the so called aleurone cell layer. During germination, the aleurone layer is activated by gibberellins, releases enzymes that digest the carbohydrates stored in the endosperm and undergoes programmed cell death ${ }^{89,90}$. The embryo is equipped with an absorptive structure called the scutellum, somewhat equivalent to human placenta, which take up the nutrients from the endosperm. In grains, a large part of the $\mathrm{Fe}$ is concentrated in the aleurone layer and another pool is found in the embryo ${ }^{69,91,92}$ (Figure 3 ). In wheat (Triticum durum), the highest concentrations of Fe are found in the aleurone layer and in the scutellum ${ }^{92}$. Separate measurements of wheat flour and bran indicated that almost 
$60 \%$ of grain $\mathrm{Fe}$ is in the bran which includes the aleurone layer and therefore represents the major $\mathrm{Fe}$ pool in grains ${ }^{93}$. Within the aleurone layer, $\mathrm{Fe}$ is concentrated together with phosphorus and other minerals in globoids inside the protein storage vacuole, similar to the subcellular localization observed in Arabidopsis ${ }^{72}$. In situ X-ray absorption spectroscopy analyses have indicated that in the aleurone layer, most Fe is bound to phytate, either as Fe(II) or $\mathrm{Fe}(\mathrm{III})$ in good agreement with its subcellular localization ${ }^{69,92}$. Another pool is bound to citrate, which may be involved in the transport from modified aleurone cells present in the crease to aleurone cells ${ }^{69}$. In rice, Fe accumulates in the aleurone layer together with phytate and in the scutellum, as observed in wheat ${ }^{91,94}$. The exact speciation of Fe in the embryo and in the endosperm has not yet been determined in these tissues that contain lower $\mathrm{Fe}$ concentration, probably because of limitations in the sensitivity of X-ray absorption spectroscopy. Based on co-occurrence of elements, it has been proposed that $\mathrm{Fe}$ is bound to phosphate (possibly as phytate) in the scutellum in wheat and to other ligands in the other parts of the embryo and in the endosperm ${ }^{69,92}$. These ligands could include proteins or smaller molecules such as NA, which is present in grains as in seeds of non graminaceous species 55,95 . In wheat flour which corresponds to the starchy endosperm, NA is the main ligand for $\mathrm{Fe}^{96}$.

Little is known on the mechanisms that control the pattern of Fe localization in grains. A developmental analysis showed that throughout rice grain development Fe co-localizes with phosphorus in the aleurone cell layer, indicating that Fe is most likely bound to phytate as soon as it is stored ${ }^{91}$. Mutations in OsVIT1, OsVIT2 and Mitochondrial Iron Transporter (MIT) have been shown to perturb iron localization within the embryo ${ }^{97,} 98$. OsYSL9 is strongly expressed in the scutellum and participates to $\mathrm{Fe}$ storage in the embryo ${ }^{53}$. The phytosiderophore efflux transporter TOM2 is expressed in the dorsal vascular bundle, epithelium and the scutellum of the embryo and may also participate in the distribution of $\mathrm{Fe}$ 19. Silencing TOM2 gene expression did not alter seed total Fe content. Analysis of $\mathrm{Fe}$ distribution using sXRF, Perls staining or seed dissection could provide more insights in its function in developing rice grains.

\section{Biofortification}

The abundance, the localization and the speciation of Fe are equally important parameters to be taken into account in attempts to increase Fe content and bioavailability in grains, which are two of the major targets of biofortification projects. Having the major Fe pool stored as Fe

372 phytate in the aleurone layer is probably the worst combination for human nutrition, as 
phytate has extremely high affinity for metals, which drastically limits $\mathrm{Fe}$ absorption in the intestine ${ }^{75}$. Moreover, the aleurone layer is most of the time discarded during grain processing, such polishing for rice or preparation of white flour for wheat ${ }^{93,96,99}$. Hence, biofortification strategies using targeted molecular approaches have aimed at changing both the localization and the speciation of Fe in grains. Several reviews have been recently published on the attempts made to biofortify wheat and rice grain ${ }^{99-101}$. This review will therefore not extensively cover the topic of biofortification and rather just highlight a few examples. The initial attempt to biofortify rice grains through biotechnology used the gene encoding ferritin, which provides $\mathrm{Fe}$ under a highly bioavailable form, driven by an endosperm specific promoter ${ }^{102}$. Although the work provided a critical proof of concept, the increase in $\mathrm{Fe}$ content was modest and variable ${ }^{103}$. Probably the most efficient strategy for biofortification by manipulating a single gene has been the activation or the over expression of NA synthase in rice grains ${ }^{95}$. This resulted not only in a strong increase in grain $\mathrm{Fe}$ concentration but also in Fe bioavailability, as NA appears to favor Fe intestinal absorption ${ }^{95}$. More recently, expression of a Fe vacuolar transporter TaVIT2 in wheat endosperm was also shown to be a relevant approach to increase Fe content and bioavailability ${ }^{70}$. Probably, the expression of TaVIT2 in the endosperm vacuoles creates a strong Fe sink during development. Subsequently, Fe becomes available when endosperm cells die and lose their internal structure. Current efforts use pyramiding of several constructs to combine the benefits of multiple transgenes. This way, by combining the expression of transporters that limit vacuolar storage with elevated ferritin and NA synthesis, an increase in Fe concentration in grains by up to 6-fold has been obtained ${ }^{104}$.

\section{A role for iron in the regulation of seed germination?}

Seed germination starts with water uptake by the dry seed and ends with radicle protrusion and initiation of cell division ${ }^{105}$. This is the critical phase of plant emergence because it is tightly regulated by water availability, temperature, oxygen, and light conditions. Germination is also controlled by endogenous factors such as the plant hormones abscisic acid (ABA), gibberellins (GA) and ethylene that play a major role in regulating early seed germination. These endogenous signals regulate germination through the process of dormancy, which is an endogenous block to the completion of germination of a mature seed ${ }^{106}$. Germination is followed by seedling growth during which mobilization of reserves and nutrient stored within the seed sustain active cell metabolism till the acquisition of autotrophy. Thus, stand 
establishment, the first critical component of crop yield, results from the successful and fast

407 completion of both germination and early seedling growth.

408 Although the role of iron in post-germinative events is quite well documented (see below) its 409 possible involvement in the regulation of germination, i.e. the sum of molecular events that 410 allow radicle protrusion, is rather unknown. The effect of iron on seed germination has mostly 411 been documented in the context of soil toxicity, since high Fe concentrations are toxic and 412 inhibit germination ${ }^{107,108}$, but this is a common feature to many metals. Increase in phytate413 degrading enzyme activity has been reported to occur during seed germination, with a 414 concomitant decline in phytate ${ }^{109-111}$ thus increasing iron bioavailability ${ }^{109}$. Müller et al. 415 (2009) showed that hydroxyl radicals were likely to play a role in cell wall loosening during 416 radicle elongation and weakening of the endosperm of cress seeds ${ }^{112}$. Although Fenton 417 reaction is a natural candidate mechanism for explaining hydroxyl radical production they did 418 not provide any evidence about its possible involvement in vivo. Thus, whether iron 419 availability participates to the numerous biochemical and molecular processes involved in the 420 regulation of radicle protrusion is actually not known.

421 Murgia and Morandini (2017) demonstrated that, when seeds developed on the mother plant 422 under Fe deficiency, they were more dormant at harvest, thus suggesting that seed $\mathrm{Fe}$ 423 availability can regulate sensu stricto germination ${ }^{113}$. It is highly likely that Fe could regulate 424 germination by controlling ROS homeostasis, through its involvement in the Fenton reaction. 425 ROS indeed have been proposed to be key players in seed germination and dormancy ${ }^{114-116 .}$ 426 Seed dormancy alleviation requires controlled ROS generation ${ }^{117}$ but excessive levels of 427 ROS trigger oxidative stress and prevent germination. This is occurring when seeds are 428 germinated in unappropriated environmental conditions or when they have aged ${ }^{115}$. With 429 regards to the relationship between Fe and ROS metabolism, we can thus hypothesize that $\mathrm{Fe}$ 430 homeostasis, as controlled by sequestration and release by vacuoles, is likely to participate in 431 the ability of seeds to germinate and to play a role in the regulation of dormancy, by buffering 432 ROS homeostasis. However whether Fe speciation (and localization) is important for seed 433 longevity and control of dormancy and germination remains to be addressed.

\section{Iron remobilization after germination}

436 Besides human nutrition, the main role of seed Fe stores is to sustain the early development of 437 seedlings after germination. In dicots in which most of the $\mathrm{Fe}$ is probably stored as Fe phytate 438 in vacuoles, retrieving Fe stores likely requires first to break down the phytate, then to 439 transport $\mathrm{Fe}$ out of the vacuole and out of the cells. In Arabidopsis, Perls DAB staining 
allowed monitoring the fate of $\mathrm{Fe}$ stores after germination: the high $\mathrm{Fe}$ concentration around vascular tissues observed in dry seeds disappeared within 4 days, indicating that Fe was rapidly redistributed the growing organs of the seedling ${ }^{79,80}$. In Arabidopsis, this process requires two redundant vacuolar transporters AtNRAMP3 and AtNRAMP4 (Natural Resistance Associated Macrophage Protein) ${ }^{82}$. AtNRAMP3 and AtNRAMP4 are highly expressed after radicle protrusion. In loss of AtNRAMP3 and AtNRAMP4 function mutants, seedling growth is arrested before the onset of photosynthesis ${ }^{82}$. In the nramp3nramp4 double mutant, Fe remains blocked inside the endodermal vacuoles after germination ${ }^{79,80,82}$. The phenotype of nramp3nramp4 is partially suppressed by mutations in AtVIT1 by redirecting Fe storage in vacuoles of cortical cells ${ }^{79}$. The phenotype of nramp3nramp 4 is also rescued by $\mathrm{Fe}$ supplementation. However, even in the presence of $\mathrm{Fe}$ in the medium, the mutant activates $\mathrm{Fe}$ deficiency responses ${ }^{118}$. Interestingly, chloroplast functions and especially Fe-requiring plastidial enzymes are repressed in the mutant while mitochondrial function is maintained ${ }^{118}$. This suggests that Fe is prioritized to mitochondria or that these organelles rely on an independent pool of Fe. AtNRAMP3 and AtNRAMP4 encode divalent cation transporters. As $\mathrm{Fe}$ is most likely stored as $\mathrm{Fe}(\mathrm{III})$ in the vacuole, it means that $\mathrm{Fe}$ reduction is probably required prior to Fe export by AtNRAMP3 and AtNRAMP4. However, the Fe reduction system active in vacuoles of germinating cells remains to be identified. The phytase allowing Fe release prior to its reduction and export also remains to be identified. The importance of such activity is highlighted by the finding that expression of the bacterial phytase US417 or mutation in the phytate biosynthetic enzyme IPK1 (Inositol Phosphate Kinase 1) in Arabidopsis significantly accelerate Fe remobilization ${ }^{119}$. After its export from the vacuole, Fe needs to be exported from storage cells (the endodermis in Arabidopsis). However, the transporters involved in Fe efflux from cells during germination have not been identified. One candidate could be IREG1/FPN1, the plant homologue of the transporter involved in the release of Fe into the blood flow from intestine epithelial cells ${ }^{30}$. Once it is exported from the cells, Fe needs to remain in a soluble form to diffuse to other cells. The citrate efflux transporter FRD3 is expressed in germinating seeds and could be involved in the release of citrate to form soluble Fe complexes in the apoplast. This hypothesis is supported by the finding that $f r d 3$ mutants are chlorotic and exhibit slow root growth after germination ${ }^{34}$. This phenotype can be rescued by supplementation of Fe or citrate in the cytosol. In relation with their distinct organization, Fe remobilization mechanisms in seeds of graminaceous species are likely very different. In grains, most of the $\mathrm{Fe}$ is in the aleurone layer, outside of the embryo. During germination, under the positive control of gibberellins, 
aleurone cells secrete amylases that digest the starch in the endosperm and eventually undergo programmed cell death (PCD) ${ }^{89}$. Membranes are destroyed in this process and it is likely that the content of globoids including Fe bound to phytate is released in the endosperm. Iron efflux from vacuoles is probably not a necessary step for mobilization. Accordingly, no orthologues of AtNRAMP3 or AtNRAMP4 have been reported in graminaceous plants. In the endosperm, phytate is probably hydrolyzed by phytase and the released Fe bound to NA. As in dicotyledonous species, the precise phytase involved have not yet been identified. Then, the main question is how is Fe taken up from the endosperm into the embryo and further distributed to growing organs within the embryo. Time course analysis of gene expression and $\mathrm{Fe}$ localization during rice germination has highlighted several candidates ${ }^{94,120}$. X-ray fluorescence imaging revealed that after $24 \mathrm{~h}$ of germination, $\mathrm{Fe}$ accumulates in the epithelium and scutellum, the Fe level decreases in the scutellum and increases in the coleoptile. Iron is already visible in the root tip after 36 hours ${ }^{94}$. Genes encoding NA and phytosiderophore biosynthesis were upregulated during germination ${ }^{120}$. Interestingly, NA synthase expression was prominent in the endosperm while NA amino transferase (NAAT) was expressed in the embryo. Accordingly, several members of the YSL NA and phytosiderophore transporters, YSL2, 6, 10, 12 and 14 are upregulated ${ }^{120}$. The expression of TOM2, a NA and phytosiderophore efflux transporter and of OsYSL2 increased markedly in the scutellum during germination ${ }^{19,120}$. These data indicate that $\mathrm{Fe}$ is transported as a complex with NA into the embryo or phytosiderophores within the embryo. Several transporters for free divalent Fe were also upregulated during germination: OsIRT1, several OsNramp genes and a Ferroportin/IREG ${ }^{94,120}$. As in dicotyledonous species, the citrate efflux transporter OsFRDL1 is upregulated indicating that citrate is also secreted to maintain Fe solubility during cell-tocell transport ${ }^{34,35}$. Finally, the rice homologue of the chloroplast Fe influx system in Arabidopsis PIC1 is upregulated after 3 days probably to sustain the differentiation of plastids into chloroplast during early seedling development ${ }^{121}$. Many transporters of $\mathrm{Fe}^{2+}$ or $\mathrm{Fe}$ complexes with NA or siderophores are apparently involved in Fe remobilization after grain germination. However, the individual contribution of each of these transporters remains to be analyzed to identify the key steps in Fe remobilization in graminaceous species.

With its uptake into chloroplasts of the developing seedling, Fe completes its journey from the senescing leaves of the mother plant to the expanding photosynthetic apparatus of its progeny, via the seed.

\section{Conclusions and perspectives}


Many transporters and ligands for Fe have been characterized and this knowledge has been used to design targeted biofortification strategies. However, many key steps remain to be elucidated at the molecular level. The mechanisms that make iron available for reallocation to the seeds during leaf senescence need to be explored in more details. It would be important to determine which of the different pathways that contribute to chloroplast degradation are most important for Fe mobilization. Important questions also still need to be answered concerning 514 phloem transport: the ligand for $\mathrm{Fe}$ in the phloem is still not unequivocally identified. Moreover, whereas OPT3 plays a key role in Fe transport to seeds, the specific substrate of this transporter is still unknown. The transfer of Fe from the mother plant to the embryo has been under investigated so far and probably deserves more attention (Figure 2). The pioneering discovery that ascorbic acid is responsible for Fe reduction in the extracellular space separating the mother tissues from the embryo raises important questions. How general this mechanism is remains to be determined. For example, the relevance of this finding for grain staples needs to be addressed. There is also a need to identify the molecular players involved in ascorbate secretion and regeneration. Moreover, even though FRD3 has been proposed to be responsible for citrate efflux in the intracellular space separating the maternal tissues and the embryo, this hypothesis would need to be substantiated and other transporters potentially involved in citrate and malate efflux need to be identified. Last but not least, the transport mechanisms responsible for Fe efflux from mother tissues and reuptake into the embryo remain completely unknown. Tissue specific transcriptomic analyses have provided candidates for mediating these steps in barley ${ }^{68}$. The role of each candidate has now to be analyzed in detail and similar analyses are lacking in Arabidopsis or other dicots. Manipulating these steps would certainly open new perspectives for biofortification. Besides the effectors of Fe transport and complexation during Fe loading into seeds, it would also be important to get insights into the master regulators that control Fe storage in seeds. How $\mathrm{Fe}$ storage is connected to the signaling pathways and transcription factors that regulate seed development, such as Leafy Cotyledon 2 (LEC2) or WRINCKLED, needs to be unraveled ${ }^{122}$. When considering Fe remobilization after germination, many questions are still open. In Arabidopsis and in dicots in general, several steps upstream and downstream of the action of vacuolar efflux by NRAMP3/4 remain to be investigated. The molecular identity of the phytases that make Fe available for transport has to be determined. Whether Fe needs to be reduced prior to transport by vacuolar NRAMP and by which mechanism are still open questions. Downstream of vacuolar efflux the plasma membrane transporters that allow $\mathrm{Fe}$ efflux from endodermal cells to other cell types, as well as the mechanisms that allow $\mathrm{Fe}$ 
542 distribution to plastids, are still to be identified. Similar questions arise concerning the use of

543 Fe stored in aleurone cells after the germination of grains of monocots. Finally, the master 544 regulators that orchestrate $\mathrm{Fe}$ remobilization in response to hormonal cues, such as 545 gibberellins, also need to be defined.

546 In conclusion, even though many players involved in Fe storage and remobilization from 547 seeds have been discovered in the last decades, we still lack a molecular understanding of 548 several key steps. In addition to improving our basic knowledge of how plants transfer 549 nutrients from one generation to the next, unraveling these steps holds great promises for seed 550 Fe biofortification and possibly for improving seed quality and stand establishment.

\section{Ackowledgements}

553 The work on seed iron storage in ST, SM and CB laboratories is supported by the CNRS, the 554 INRA and Paris Sorbonne University, as well as by the collaborative ANR grant ISISTOR 555 (ANR-16-CE20-0019-02).

\section{References}

1. Have, M., et al., (2017)Nitrogen remobilization during leaf senescence: lessons from Arabidopsis to crops. J Exp Bot. 68(10): p. 2513-2529.

2. Pottier, M., et al., (2019)Autophagy is essential for optimal translocation of iron to seeds in Arabidopsis. J Exp Bot. 70(3): p. 859-869.

3. Waters, B.M. and M.A. Grusak, (2008)Whole-plant mineral partitioning throughout the life cycle in Arabidopsis thaliana ecotypes Columbia, Landsberg erecta, Cape Verde Islands, and the mutant line ysl1ysl3. New Phytol. 177(2): p. 389-405.

4. Murgia, I., et al., (2012)Biofortification for combating 'hidden hunger' for iron. Trends Plant Sci. 17(1): p. 47-55.

5. Grillet, L., S. Mari, and W. Schmidt, (2014)Iron in seeds - loading pathways and subcellular localization. Front Plant Sci. 4: p. 535.

6. Connorton, J.M., J. Balk, and J. Rodriguez-Celma, (2017)Iron homeostasis in plants - a brief overview. Metallomics. 9(7): p. 813-823.

7. Kobayashi, T. and N.K. Nishizawa, (2012)Iron uptake, translocation, and regulation in higher plants. Annu Rev Plant Biol. 63: p. 131-52.

8. Santi, S. and W. Schmidt, (2009)Dissecting iron deficiency-induced proton extrusion in Arabidopsis roots. New Phytol. 183(4): p. 1072-84.

9. Rajniak, J., et al., (2018)Biosynthesis of redox-active metabolites in response to iron deficiency in plants. Nat Chem Biol. 14(5): p. 442-450.

10. Tsai, H.H. and W. Schmidt, (2017)Mobilization of Iron by Plant-Borne Coumarins. Trends Plant Sci. 22(6): p. 538-548.

11. Robinson, N.J., et al., (1999)A ferric-chelate reductase for iron uptake from soils. Nature (London). 397(6721): p. 694-697.

12. Vert, G., et al., (2002)IRT1, an Arabidopsis transporter essential for iron uptake from the soil and for plant growth. Plant Cell. 14(6): p. 1223-33.

13. Connolly, E.L., J.P. Fett, and M.-L. Guerinot, (2002)Expression of the IRT1 metal transporter is controlled by metals at the levels of transcript and protein accumulation. The Plant Cell. 14: p. 1347-1357. 
14. Eide, D., et al., (1996)A novel iron-regulated metal transporter from plants identified by functional expression in yeast. Proceedings of the National Academy of Sciences of the United States of America. 93(11): p. 5624-5628.

15. Korshunova, Y.O., et al., (1999)The IRT1 protein from Arabidopsis thaliana is a metal transporter with a broad substrate range. Plant Molecular Biology. 40(1): p. 37-44.

16. Dubeaux, G., et al., (2018)Metal Sensing by the IRT1 Transporter-Receptor Orchestrates Its Own Degradation and Plant Metal Nutrition. Mol Cell. 69(6): p. 953-964 e5.

17. Thomine, S. and G. Vert, (2013)Iron transport in plants: better be safe than sorry. Curr Opin Plant Biol. 16(3): p. 322-7.

18. Chan-Rodriguez, D. and E.L. Walker, (2018)Analysis of Yellow Striped Mutants of Zea mays Reveals Novel Loci Contributing to Iron Deficiency Chlorosis. Front Plant Sci. 9: p. 157.

19. Nozoye, T., et al., (2015)The Phytosiderophore Efflux Transporter TOM2 Is Involved in Metal Transport in Rice. J Biol Chem. 290(46): p. 27688-99.

20. Curie, C., et al., (2001)Maize yellow stripe1 encodes a membrane protein directly involved in $\mathrm{Fe}(\mathrm{III})$ uptake. Nature. 409(6818): p. 346-349.

21. Inoue, H., et al., (2009)Rice OsYSL15 is an iron-regulated iron(III)-deoxymugineic acid transporter expressed in the roots and is essential for iron uptake in early growth of the seedlings. J Biol Chem. 284(6): p. 3470-9.

22. Lee, S., et al., (2009)Disruption of OsYSL15 leads to iron inefficiency in rice plants. Plant Physiol. 150(2): p. 786-800.

23. Gao, F., et al., (2019)The Transcriptional Control of Iron Homeostasis in Plants: A Tale of bHLH Transcription Factors? Front Plant Sci. 10: p. 6.

24. Kobayashi, T., T. Nozoye, and N.K. Nishizawa, (2019)Iron transport and its regulation in plants. Free Radic Biol Med. 133: p. 11-20.

25. Kobayashi, T., et al., (2013)Iron-binding haemerythrin RING ubiquitin ligases regulate plant iron responses and accumulation. Nat Commun. 4: p. 2792.

26. Long, T.A., et al., (2010)The bHLH transcription factor POPEYE regulates response to iron deficiency in Arabidopsis roots. Plant Cell. 22(7): p. 2219-36.

27. Rodriguez-Celma, J., et al., (2019)Arabidopsis BRUTUS-LIKE E3 ligases negatively regulate iron uptake by targeting transcription factor FIT for recycling. Proc Natl Acad Sci U S A.

28. Barberon, M., (2017)The endodermis as a checkpoint for nutrients. New Phytol. 213(4): p. 1604-1610.

29. Barberon, M., et al., (2016)Adaptation of Root Function by Nutrient-Induced Plasticity of Endodermal Differentiation. Cell. 164(3): p. 447-59.

30. Morrissey, J., et al., (2009)The ferroportin metal efflux proteins function in iron and cobalt homeostasis in Arabidopsis. Plant Cell. 21(10): p. 3326-38.

31. Durrett, T.P., W. Gassmann, and E.E. Rogers, (2007)The FRD3-mediated efflux of citrate into the root vasculature is necessary for efficient iron translocation. Plant Physiol. 144(1): p. 197205.

32. Flis, P., et al., (2016)Inventory of metal complexes circulating in plant fluids: a reliable method based on HPLC coupled with dual elemental and high-resolution molecular mass spectrometric detection. New Phytol. 211(3): p. 1129-41.

33. Rogers, E.E. and M.L. Guerinot, (2002)FRD3, a member of the multidrug and toxin efflux family, controls iron deficiency responses in Arabidopsis. Plant Cell. 14(8): p. 1787-99.

34. Roschzttardtz, H., et al., (2011)The FRD3 citrate effluxer promotes iron nutrition between symplastically disconnected tissues throughout Arabidopsis development. Plant Cell. 23(7): $p$. 2725-37.

35. Yokosho, K., et al., (2009)OsFRDL1 is a citrate transporter required for efficient translocation of iron in rice. Plant Physiol. 149(1): p. 297-305.

36. Lanquar, V., et al., (2010)Export of vacuolar manganese by AtNRAMP3 and AtNRAMP4 is required for optimal photosynthesis and growth under manganese deficiency. Plant Physiol. 152(4): p. 1986-99. 
37. Roschzttardtz, H., et al., (2013)New insights into Fe localization in plant tissues. Front Plant Sci. 4: p. 350.

38. Shingles, R., M. North, and R.E. McCarty, (2002)Ferrous ion transport across chloroplast inner envelope membranes. Plant Physiol. 128(3): p. 1022-30.

39. Pottier, M., et al., (2014)Autophagy as a possible mechanism for micronutrient remobilization from leaves to seeds. Front Plant Sci. 5: p. 11.

40. Guiboileau, A., et al., (2012)Autophagy machinery controls nitrogen remobilization at the whole-plant level under both limiting and ample nitrate conditions in Arabidopsis. New Phytol. 194(3): p. 732-40.

41. Otegui, M.S., (2018)Vacuolar degradation of chloroplast components: autophagy and beyond. J Exp Bot. 69(4): p. 741-750.

42. Izumi, M., et al., (2017)Entire Photodamaged Chloroplasts Are Transported to the Central Vacuole by Autophagy. Plant Cell. 29(2): p. 377-394.

43. Michaeli, S., et al., (2014)Arabidopsis ATG8-INTERACTING PROTEIN1 is involved in autophagydependent vesicular trafficking of plastid proteins to the vacuole. Plant Cell. 26(10): p. 4084101.

44. Wang, S. and E. Blumwald, (2014)Stress-induced chloroplast degradation in Arabidopsis is regulated via a process independent of autophagy and senescence-associated vacuoles. Plant Cell. 26(12): p. 4875-88.

45. Qiu, K., et al., (2015)EIN3 and ORE1 Accelerate Degreening during Ethylene-Mediated Leaf Senescence by Directly Activating Chlorophyll Catabolic Genes in Arabidopsis. PLoS Genet. 11(7): p. e1005399.

46. Ricachenevsky, F.K., P.K. Menguer, and R.A. Sperotto, (2013)kNACking on heaven's door: how important are NAC transcription factors for leaf senescence and Fe/Zn remobilization to seeds? Front Plant Sci. 4: p. 226.

47. Uauy, C., et al., (2006)A NAC Gene regulating senescence improves grain protein, zinc, and iron content in wheat. Science. 314(5803): p. 1298-301.

48. Klatte, M., et al., (2009)The analysis of Arabidopsis nicotianamine synthase mutants reveals functions for nicotianamine in seed iron loading and iron deficiency responses. Plant Physiol. 150(1): p. 257-71.

49. Ling, H.Q., et al., (1999)Map-based cloning of chloronerva, a gene involved in iron uptake of higher plants encoding nicotianamine synthase. Proceedings of the National Academy of Sciences of the United States of America. 96: p. 7098-103.

50. Takahashi, M., et al., (2003)Role of nicotianamine in the intracellular delivery of metals and plant reproductive development. Plant Cell. 15(6): p. 1263-80.

51. Koike, S., et al., (2004)OsYSL2 is a rice metal-nicotianamine transporter that is regulated by iron and expressed in the phloem. Plant J. 39(3): p. 415-24.

52. Ishimaru, Y., et al., (2010)Rice metal-nicotianamine transporter, OsYSL2, is required for the long-distance transport of iron and manganese. Plant J. 62(3): p. 379-90.

53. Senoura, T., et al., (2017)The iron-chelate transporter OsYSL9 plays a role in iron distribution in developing rice grains. Plant Mol Biol. 95(4-5): p. 375-387.

54. Chu, H.H., et al., (2010)Successful reproduction requires the function of Arabidopsis Yellow Stripe-Like1 and Yellow Stripe-Like3 metal-nicotianamine transporters in both vegetative and reproductive structures. Plant Physiol. 154(1): p. 197-210.

55. Le Jean, M., et al., (2005)A loss-of-function mutation in AtYSL1 reveals its role in iron and nicotianamine seed loading. Plant J. 44(5): p. 769-82.

56. Waters, B.M., et al., (2006)Mutations in Arabidopsis yellow stripe-like1 and yellow stripelike3 reveal their roles in metal ion homeostasis and loading of metal ions in seeds. Plant Physiol. 141(4): p. 1446-58.

57. Schuler, M., et al., (2012)Nicotianamine functions in the Phloem-based transport of iron to sink organs, in pollen development and pollen tube growth in Arabidopsis. Plant Cell. 24(6): p. $2380-400$. 
58. Stacey, M.G., et al., (2002)AtOPT3, a member of the oligopeptide transporter family, is essential for embryo development in Arabidopsis. Plant Cell. 14(11): p. 2799-811.

59. Bogs, J., et al., (2003)Functional characterization and expression analysis of a glutathione transporter, BjGT1, from Brassica juncea: evidence for regulation by heavy metal exposure. Plant Cell Environ. 26: p. 1703-1711.

60. Zhai, Z., et al., (2014)OPT3 Is a Phloem-Specific Iron Transporter That Is Essential for Systemic Iron Signaling and Redistribution of Iron and Cadmium in Arabidopsis. Plant Cell. 26(5): p. 2249-2264.

61. Stacey, M.G., et al., (2008)The Arabidopsis AtOPT3 protein functions in metal homeostasis and movement of iron to developing seeds. Plant Physiol. 146(2): p. 589-601.

62. Mendoza-Cozatl, D.G., et al., (2014)OPT3 is a component of the iron-signaling network between leaves and roots and misregulation of OPT3 leads to an over-accumulation of cadmium in seeds. Mol Plant. 7(9): p. 1455-1469.

63. Bashir, K., et al., (2015)Iron deficiency regulated OsOPT7 is essential for iron homeostasis in rice. Plant Mol Biol. 88(1-2): p. 165-76.

64. Grillet, L., et al., (2014)Ascorbate efflux as a new strategy for iron reduction and transport in plants. J Biol Chem. 289(5): p. 2515-25.

65. Jain, A., G.T. Wilson, and E.L. Connolly, (2014)The diverse roles of FRO family metalloreductases in iron and copper homeostasis. Front Plant Sci. 5: p. 100.

66. Jeong, J., et al., (2008)Chloroplast Fe(III) chelate reductase activity is essential for seedling viability under iron limiting conditions. Proc Natl Acad Sci U S A. 105(30): p. 10619-24.

67. Olsen, L.I., et al., (2016)Mother-plant-mediated pumping of zinc into the developing seed. Nat Plants. 2(5): p. 16036.

68. Borg, S., et al., (2009)Iron Transport, deposition and bioavailability in the wheat and barley grain. Plant Soil. 325: p. 15-24.

69. De Brier, N., et al., (2016)Element distribution and iron speciation in mature wheat grains (Triticum aestivum L.) using synchrotron X-ray fluorescence microscopy mapping and X-ray absorption near-edge structure (XANES) imaging. Plant Cell Environ. 39(8): p. 1835-47.

70. Connorton, J.M., et al., (2017)Wheat Vacuolar Iron Transporter TaVIT2 Transports Fe and Mn and Is Effective for Biofortification. Plant Physiol. 174(4): p. 2434-2444.

71. Kim, S.A., et al., (2006)Localization of iron in Arabidopsis seed requires the vacuolar membrane transporter VIT1. Science. 314(5803): p. 1295-8.

72. Lott, J.N. and E. Spitzer, (1980)X-ray Analysis Studies of Elements Stored in Protein Body Globoid Crystals of Triticum Grains. Plant Physiol. 66(3): p. 494-9.

73. Curie, C., et al., (2009)Metal movement within the plant: contribution of nicotianamine and yellow stripe 1-like transporters. Ann Bot. 103(1): p. 1-11.

74. Clemens, S., (2014)Zn and Fe biofortification: the right chemical environment for human bioavailability. Plant Sci. 225: p. 52-7.

75. Hallberg, L., (2001)Perspective on nutritional iron deficiency. Annual Review of Nutrition. 21: p. 1-21.

76. Briat, J.F., (1999)Plant ferritin and human iron deficiency. Nat Biotechnol. 17(7): p. 621.

77. Chu, H.H., et al., (2017)The Arabidopsis MTP8 transporter determines the localization of manganese and iron in seeds. Sci Rep. 7(1): p. 11024.

78. Eroglu, S., et al., (2017)Metal Tolerance Protein 8 Mediates Manganese Homeostasis and Iron Reallocation during Seed Development and Germination. Plant Physiol. 174(3): p. 16331647.

79. Mary, V., et al., (2015)Bypassing Iron Storage in Endodermal Vacuoles Rescues the Iron Mobilization Defect in the natural resistance associated-macrophage protein3natural resistance associated-macrophage protein4 Double Mutant. Plant Physiol. 169(1): p. 748-59.

80. Roschzttardtz, H., et al., (2009)Identification of the endodermal vacuole as the iron storage compartment in the Arabidopsis embryo. Plant Physiol. 151(3): p. 1329-38. 
81. Schnell Ramos, M., et al., (2013)Using muPIXE for quantitative mapping of metal concentration in Arabidopsis thaliana seeds. Front Plant Sci. 4: p. 168.

82. Lanquar, V., et al., (2005)Mobilization of vacuolar iron by AtNRAMP3 and AtNRAMP4 is essential for seed germination on low iron. EMBO J. 24(23): p. 4041-51.

83. Ibeas, M.A., et al., (2017)Dynamic Subcellular Localization of Iron during Embryo Development in Brassicaceae Seeds. Front Plant Sci. 8: p. 2186.

84. Roschzttardtz, H., et al., (2011)Plant cell nucleolus as a hot spot for iron. J Biol Chem. 286(32): p. 27863-6.

85. Moore, K.L., et al., (2018)The stage of seed development influences iron bioavailability in pea (Pisum sativum L.). Sci Rep. 8(1): p. 6865.

86. Cvitanich, C., et al., (2010)Iron and ferritin accumulate in separate cellular locations in Phaseolus seeds. BMC Plant Biol. 10: p. 26.

87. Eroglu, S., et al., (2019)The Conservation of VIT1-Dependent Iron Distribution in Seeds. Front Plant Sci. 10: p. 907.

88. Ibeas, M.A., et al., (2018)The Diverse Iron Distribution in Eudicotyledoneae Seeds: From Arabidopsis to Quinoa. Front Plant Sci. 9: p. 1985.

89. Fath, A., P.C. Bethke, and R.L. Jones, (1999)Barley aleurone cell death is not apoptotic: characterization of nuclease activities and DNA degradation. Plant J. 20(3): p. 305-15.

90. Jones, R.L., (1969)Gibberellic acid and the fine structure of barley aleurone cells : II. Changes during the synthesis and secretion of alpha-amylase. Planta. 88(1): p. 73-86.

91. Iwai, T., et al., (2012)Dynamic changes in the distribution of minerals in relation to phytic acid accumulation during rice seed development. Plant Physiol. 160(4): p. 2007-14.

92. Singh, S.P., et al., (2013)Pattern of iron distribution in maternal and filial tissues in wheat grains with contrasting levels of iron. J Exp Bot. 64(11): p. 3249-60.

93. De Brier, N., et al., (2015)Distribution of Minerals in Wheat Grains (Triticum aestivum L.) and in Roller Milling Fractions Affected by Pearling. J Agric Food Chem. 63(4): p. 1276-1285.

94. Takahashi, M., et al., (2009)In vivo analysis of metal distribution and expression of metal transporters in rice seed during germination process by microarray and $\mathrm{X}$-ray Fluorescence Imaging of $\mathrm{Fe}, \mathrm{Zn}, \mathrm{Mn}$, and $\mathrm{Cu}$. Plant and Soil. 325: p. 39-51.

95. Lee, S., et al., (2009)Iron fortification of rice seeds through activation of the nicotianamine synthase gene. Proc Natl Acad Sci U S A. 106(51): p. 22014-9.

96. Eagling, T., et al., (2014)Distribution and speciation of iron and zinc in grain of two wheat genotypes. J Agric Food Chem. 62(3): p. 708-16.

97. Bashir, K., et al., (2013)The knockdown of OsVIT2 and MIT affects iron localization in rice seed. Rice (N Y). 6(1): p. 31.

98. Zhang, Y., et al., (2012)Vacuolar membrane transporters OsVIT1 and OsVIT2 modulate iron translocation between flag leaves and seeds in rice. Plant J. 72(3): p. 400-10.

99. Kawakami, Y. and N.K. Bhullar, (2018)Molecular processes in iron and zinc homeostasis and their modulation for biofortification in rice. J Integr Plant Biol. 60(12): p. 1181-1198.

100. Bashir, K., et al., (2013)Exploiting new tools for iron bio-fortification of rice. Biotechnol Adv. 31(8): p. 1624-33.

101. Connorton, J.M. and J. Balk, (2019)Iron Biofortification of Staple Crops: Lessons and Challenges in Plant Genetics. Plant Cell Physiol. 60(7): p. 1447-1456.

102. Goto, F., et al., (1999)Iron fortification of rice seed by the soybean ferritin gene. Nat Biotechnol. 17(3): p. 282-6.

103. Qu le, Q., et al., (2005)Iron accumulation does not parallel the high expression level of ferritin in transgenic rice seeds. Planta. 222(2): p. 225-33.

104. Wu, T.Y., W. Gruissem, and N.K. Bhullar, (2019)Targeting intracellular transport combined with efficient uptake and storage significantly increases grain iron and zinc levels in rice. Plant Biotechnol J. 17(1): p. 9-20.

105. Bewley, J.D., (1997)Seed Germination and Dormancy. Plant Cell. 9(7): p. 1055-1066. 
106. Finch-Savage, W.E. and G. Leubner-Metzger, (2006)Seed dormancy and the control of germination. New Phytol. 171(3): p. 501-23.

107. Li, X., et al., (2012)Responses of seedling growth and antioxidant activity to excess iron and copper in Triticum aestivum L. Ecotoxicol Environ Saf. 86: p. 47-53.

108. Nozoe, T., et al., (2009)Effects of ferrous iron (Fe) on the germination and root elongation of paddy rice and weeds. Weed Biol. Manag. . 9: p. 20-26.

109. Afify Ael, M., et al., (2011)Bioavailability of iron, zinc, phytate and phytase activity during soaking and germination of white sorghum varieties. PLoS One. 6(10): p. e25512.

110. Azeke, M.A., et al., (2011)Effect of germination on the phytase activity, phytate and total phosphorus contents of rice (Oryza sativa), maize (Zea mays), millet (Panicum miliaceum), sorghum (Sorghum bicolor) and wheat (Triticum aestivum). J Food Sci Technol. 48(6): p. 7249.

111. Tian, B., et al., (2010)Physicochemical changes of oat seeds during germination. Food Chemistry. 119: p. 1195-1200.

112. Müller, K., et al., ((2009)In Vivo Cell Wall Loosening by Hydroxyl Radicals during Cress Seed Germination and Elongation Growth. Plant Physiol. 150: p. 1855-1865.

113. Murgia, I. and P. Morandini, (2017)Iron Deficiency Prolongs Seed Dormancy in Arabidopsis Plants. Front Plant Sci. 8: p. 2077.

114. Bailly, C., (2004) Active oxygen species and antioxidants in seed biology. Seed Science Research. 14: p. 93-107.

115. Bailly, C., H. El-Maarouf-Bouteau, and F. Corbineau, (2008)From intracellular signaling networks to cell death: the dual role of reactive oxygen species in seed physiology. C R Biol. 331(10): p. 806-14.

116. El-Maarouf-Bouteau, H., et al., (2015)Reactive oxygen species, abscisic acid and ethylene interact to regulate sunflower seed germination. Plant Cell Environ. 38(2): p. 364-74.

117. Leymarie, J., et al., (2012)Role of reactive oxygen species in the regulation of Arabidopsis seed dormancy. Plant Cell Physiol. 53(1): p. 96-106.

118. Bastow, E.L., et al., (2018)Vacuolar Iron Stores Gated by NRAMP3 and NRAMP4 Are the Primary Source of Iron in Germinating Seeds. Plant Physiol. 177(3): p. 1267-1276.

119. Belgaroui, N., et al., (2014)Over-expression of the bacterial phytase US417 in Arabidopsis reduces the concentration of phytic acid and reveals its involvement in the regulation of sulfate and phosphate homeostasis and signaling. Plant Cell Physiol. 55(11): p. 1912-24.

120. Nozoye, T., et al., (2007)The expression of iron homeostasis-related genes during rice germination. Plant Mol Biol. 64(1-2): p. 35-47.

121. Duy, D., et al., (2007)PIC1, an ancient permease in Arabidopsis chloroplasts, mediates iron transport. Plant Cell. 19(3): p. 986-1006.

122. Baud, S., et al., (2016)Deciphering the Molecular Mechanisms Underpinning the Transcriptional Control of Gene Expression by Master Transcriptional Regulators in Arabidopsis Seed. Plant Physiol. 171(2): p. 1099-112. 


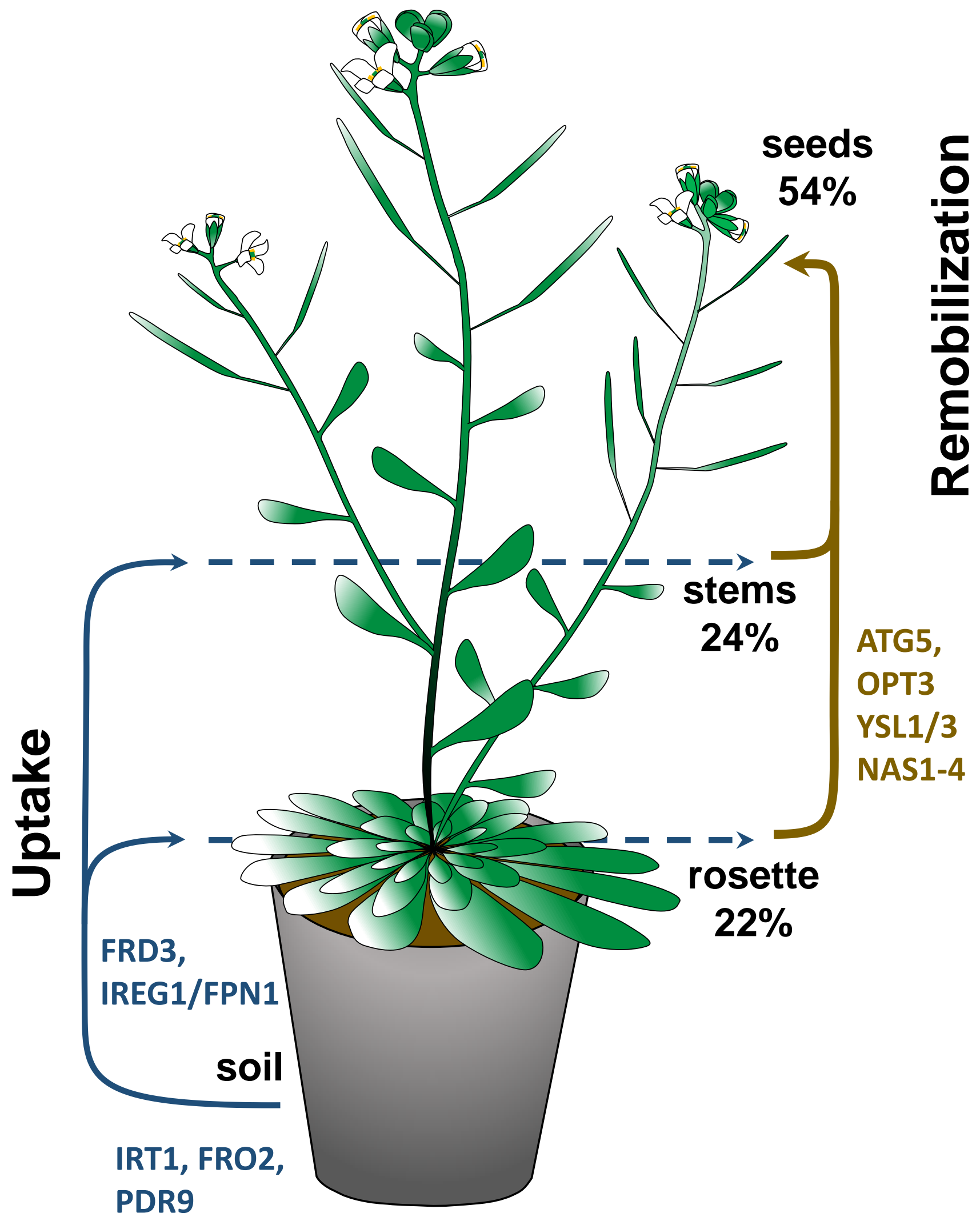

Figure 1: Fe uptake and remobilization to seeds in Arabidopsis thaliana. The names of the main proteins involved in uptake and translocation from roots to shoots are indicated in blue. The name of the main proteins involved in remobilization to seeds are indicated in brown. The percentages indicate the mean distribution of $\mathrm{Fe}$ at the end of the plant's life cycle, excluding the roots (adapted from Pottier et al., 2019) 

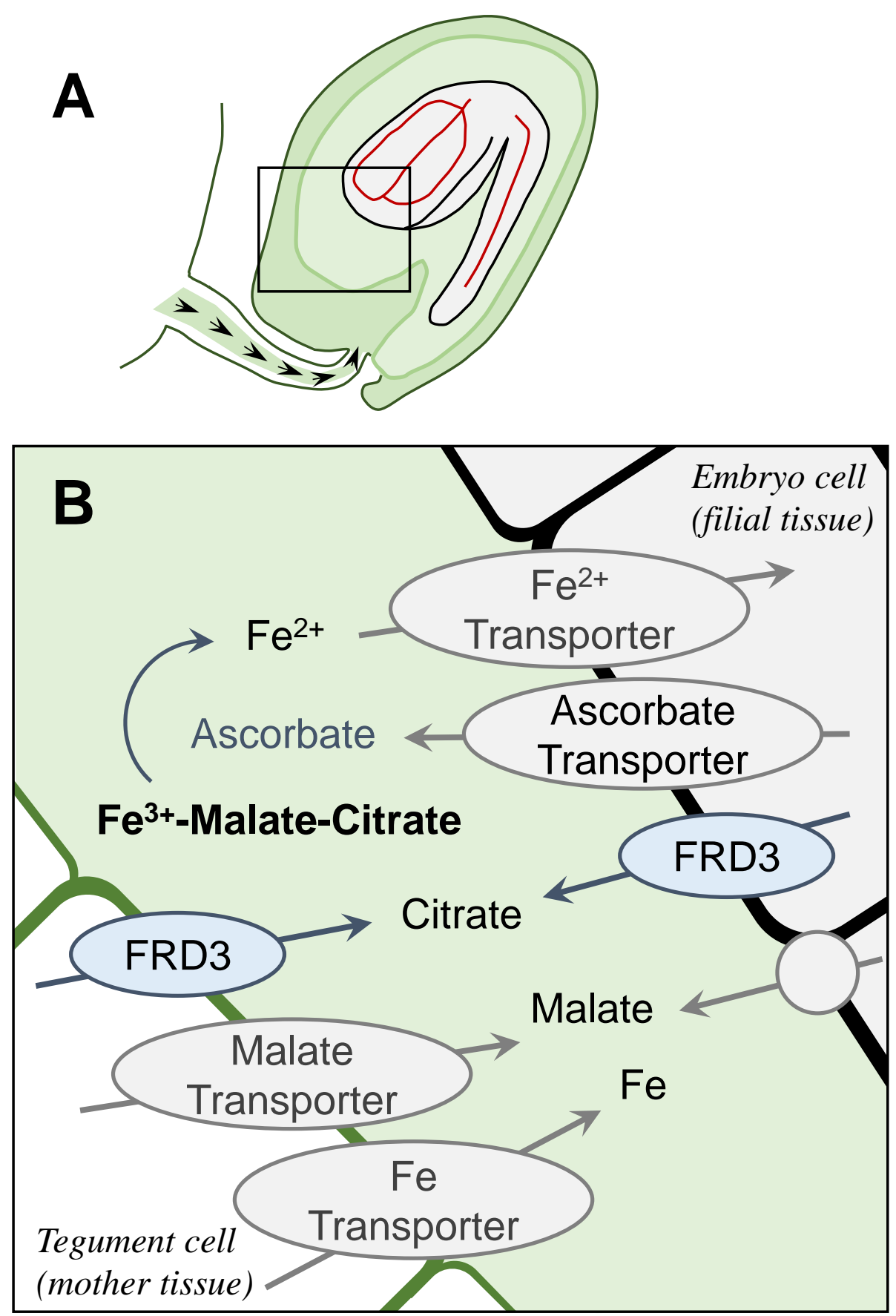

Figure 2: Fe transfer from the maternal to embryo tissues. A. Fe is transported to the maternal tissues of the developing seed but has to cross an extracelluar space between the teguments and the embryo. B. Fe is bound to malate and citrate in the extracellular space separating the maternal tissues from the embryo. In Arabidopsis and pea, Fe is reduced by ascorbate prior to its uptake into the embryo. The transporters responsible for $\mathrm{Fe}$, malate and ascorbate secretion to the extracellular space as well as the transporter responsible for Fe uptake into the embryo remain to be identified. 


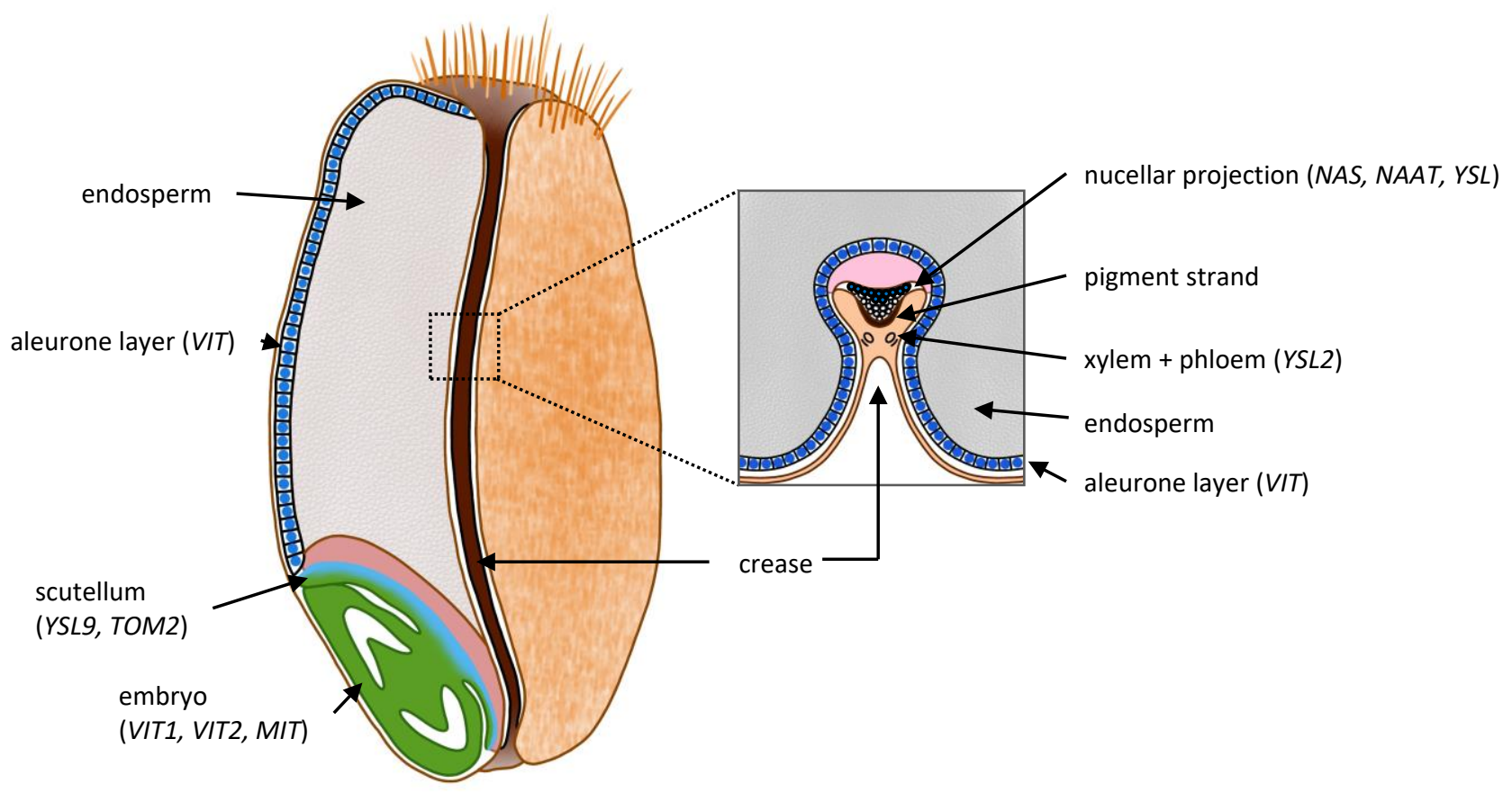

Figure 3: Fe distribution in a mature cereal grain (eg wheat grain). Fe-rich tissues and cells appear in blue, symplastic disconnections (between maternal tissues and endosperm; between endosperm and scutellum) are highlighted in pink. The size, shape and development of the crease and the nucellar projection vary among the monocot phylogeny. 

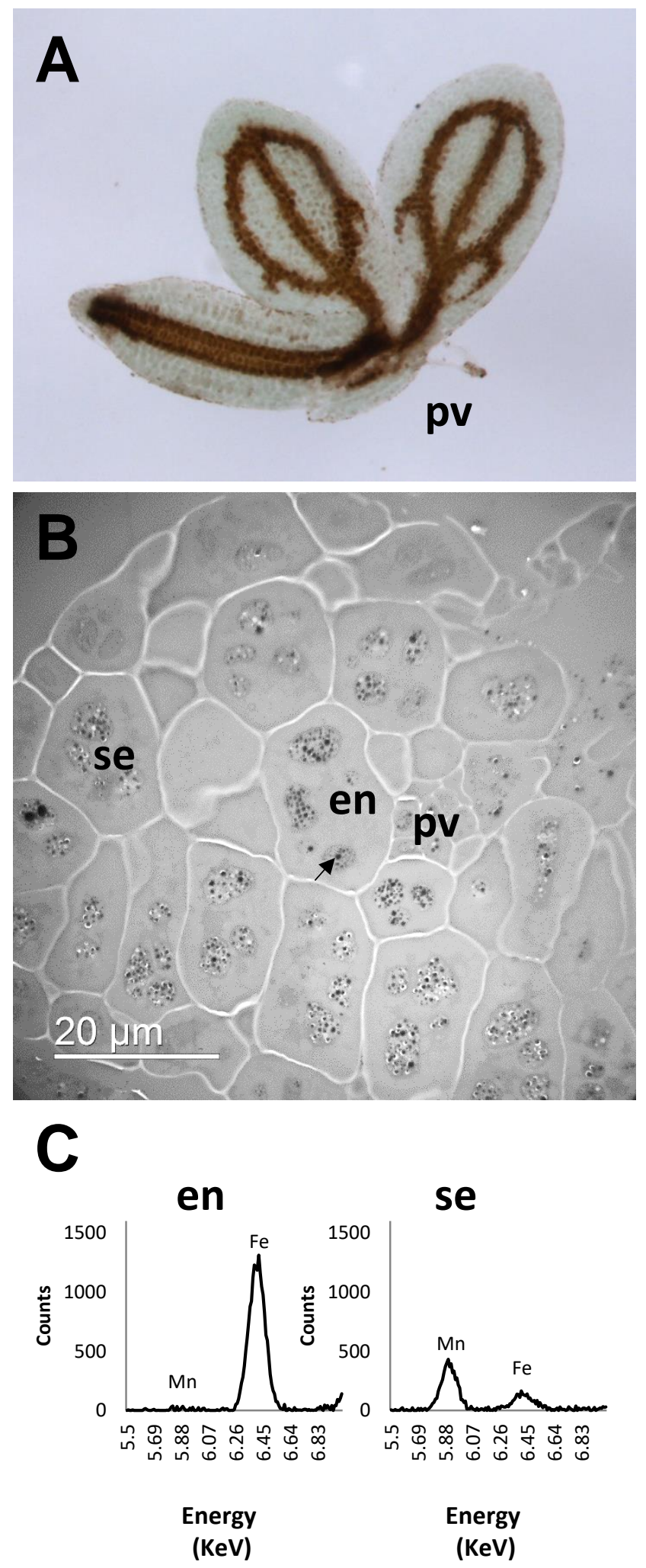

Figure 4: Fe distribution in Arabidopsis thaliana mature embryo. A. Perls DAB staining of $\mathrm{Fe}$ in the whole embryo shows high concentration of $\mathrm{Fe}$ (brown color) around the provasculature. B. TEM micrograph of an embryo section in the cotyledon showing the provasculature (pv), proto-endodermis cells (en) an subepidermal cells (se). The phytate globoids appear as small black dots inside the storage vacuole (arrow). C. EDX analysis of protein bodies in proto-endodermal (en) or sub-epidermal cells (se): Fe is concentrated in the storage vacuole of proto-endodermal cells (en). 